\title{
SOLVED AND UNSOLVED PROBLEMS ON POLYNOMIALS
}

\author{
ANDRZEJ SCHINZEL \\ Institute of Mathematics, Polish Academy of Sciences \\ Śniadeckich 8, 00-950 Warszawa, Poland \\ E-mail: schinzel@impan.gov.pl
}

We shall tell about problems concerning polynomials in one variable over an arbitrary field $K$ considered during the last hundred years.

In 1895 Vahlen proved for the rational field $\mathbb{Q}$ the following theorem, which in 1897 was extended by Capelli [1] to all fields of characteristic 0 .

CApelli's Theorem. A binomial $x^{n}-a$ is reducible over a field $K$ if and only if either $a=b^{p}, p$ prime, $p \mid n, b \in K$ or $a=-4 b^{4}, 4 \mid n, b \in K$.

(The theorem also is true for fields of positive characteristic as shown by Rédei [11]).

COROllary. Every binomial over $\mathbb{Q}$ has at least one irreducible factor with at most three non-zero coefficients.

R e m a r k. The equality $x^{4}+4=\left(x^{2}-2 x+2\right)\left(x^{2}+2 x+2\right)$ shows that the number three in the corollary is best possible.

In 1922 Ritt [13] introduced the following

Definition. Let $f \in \mathbb{C}[x]$. The polynomial $f$ is called prime, if its degree $\partial f>1$ and

$$
f=g(h) \Rightarrow \partial g=1 \text { or } \partial h=1 \text {. }
$$

Of course, every polynomial is a composition (o) of prime polynomials. Is the decomposition unique? The question should be made precise. First of all, for

1991 Mathematics Subject Classification: 12.

Lecture given at the Banach Center Colloquium on 25th February 1993.

The paper is in final form and no version of it will be published elsewhere. 
a linear polynomial $l$ we have

$$
g \circ h=(g \circ l) \circ\left(l^{-1} \circ h\right) .
$$

Next, the composition of polynomials in not, in general, commutative. On the other hand, we have the following example

$$
\begin{array}{ll}
g_{1}=x^{v} p(x)^{n}, & h_{1}=x^{n}, \\
g_{2}=x^{n}, & h_{2}=x^{v} p\left(x^{n}\right) .
\end{array}
$$

For the above polynomials we have $g_{1} \circ h_{1}=g_{2} \circ h_{2}$ and we can choose a polynomial $p$ so that the polynomials $g_{1}, h_{1}, g_{2}, h_{2}$ are prime, thus there is no uniqueness of decomposition.

The two theorems given below were proved by Ritt for polynomials over $\mathbb{C}$.

First RitT's TheOREM. If polynomials $f_{i}$ an $g_{j}$ for $1 \leq i \leq r, 1 \leq j \leq s$ are prime and

$$
f=f_{1} \circ f_{2} \circ \ldots \circ f_{r}=g_{1} \circ g_{2} \ldots \circ g_{s},
$$

then $r=s$ and the vector $\left[\partial f_{1}, \ldots, \partial f_{r}\right]$ is a permutation of $\left[\partial g_{1}, \ldots, \partial g_{s}\right]$.

Ritt's proof was analytic, using Riemann surfaces. In 1969 Fried and MacRae [7] gave an algebraic proof valid for an arbitrary field $K$ under the assumption that $\partial f \not \equiv 0 \bmod$ char $K$, reducing the theorem to the Jordan-Hölder theorem about finite groups. In 1974 Dorey and Whaples [4] showed that without the above assumption the theorem is not true in general. Much deeper is second Ritt's theorem.

SECOND RitT's TheOREM. If $g_{1} \circ h_{1}=g_{2} \circ h_{2}, \partial g_{1}=\partial h_{2}=m>\partial h_{1}=$ $\partial g_{2}=n,(m, n)=1$ then up to transformations by linear functions we either have (1) or $g_{1}=D_{m}=h_{2}, g_{2}=D_{n}=h_{1}$, where the polynomials $D_{k}$ are given by the formula

$$
D_{k}\left(x+x^{-1}\right)=x^{k}+x^{-k} .
$$

The author in his lectures on polynomials [15] presented a proof of this theorem for polynomials over an algebraically closed field $K$ satisfying the condition

$$
\operatorname{char} K=0 \quad \text { or } \quad \operatorname{char} K>\max \{m, n\}
$$

and indicated the changes necessary if $K$ is not algebraically closed. Recently Zannier [21] has proved the above theorem for algebraically closed $K$ without condition (2). He has assumed only that $g_{1}^{\prime} g_{2}^{\prime} h_{1}^{\prime} h_{2}^{\prime} \not \equiv 0$.

In 1933 D. H. Lehmer [9] asked (in an equivalent formulation) the following question. Let $f \in \mathbb{Z}[x]$ be monic and assume that $f(0) \neq 0$ and $f$ is not a product of cyclotomic polynomials. Does there exist a constant $C>1$ (independent of $f$ ) such that

$$
M(f)=\prod_{f(\xi)=0} \max \{1,|\xi|\} \geq C
$$

(multiple zeros counted multiply)? 
Lehmer indicated a possible value of $C$, as the unique real greater than 1 root of the equation

$$
f_{0}(x)=x^{10}+x^{9}-x^{7}-x^{6}-x^{5}-x^{4}-x^{3}+x+1=0,
$$

approximately equal to 1.17 . One sees that $f_{0}$ is a reciprocal polynomial, i.e.

$$
f_{0}\left(x^{-1}\right)= \pm x^{-\partial f_{0}} f_{0}(x) .
$$

This is not an accident, since Smyth proved in [19] that if $f$ is not reciprocal then $M(f) \geq \vartheta_{0}$, where $\vartheta \approx 1.32$ is a unique real root of $x^{3}-x-1=0$.

For reciprocal polynomials an essential progress was achieved by E. Dobrowolski, who proved in [3] that for all $\varepsilon>0$ and $\partial f \geq n_{0}(\varepsilon)$

$$
M(f) \geq 1+(c-\varepsilon)\left(\frac{\log \log \partial f}{\log \partial f}\right)^{3},
$$

where $c=1$.

The value of $c$ has been improved. The present record belongs to R. Louboutin [10] and is $c=9 / 4$.

Lehmer's problem is related to the following problem proposed in [18] by Zassenhaus and the author. Is it true under the same conditions on $f$ as before that

$$
\max _{f(\xi)=0}|\xi|>1+\frac{C}{\partial f},
$$

where $C>0$ is an absolute constant?

Combining the theorems of Smyth and Louboutin we infer that

$$
\max _{f(\xi)=0}|\xi|>1+\left(\frac{9}{2}-\varepsilon\right) \frac{1}{\partial f}\left(\frac{\log \log \partial f}{\log \partial f}\right)^{3} .
$$

Recently, A. Dubickas [5] has proved that

$$
\max _{f(\xi)=0}|\xi|>1+\left(\frac{64}{\pi^{2}}-\varepsilon\right) \frac{1}{\partial f}\left(\frac{\log \log \partial f}{\log \partial f}\right)^{3} .
$$

Let us return now to the chronological order of problems. In 1947 Rényi [12] found an example of a polynomial $f \in \mathbb{Q}[x]$ complete, i.e. with all coefficients different from zero, of degree 28 and such that $f^{2}$ has only 28 non-zero coefficients.

Denoting the number of non-zero coefficients of a polynomial $g$ by $N(g)$ we may conclude from Rényi's example that there exist $f \in \mathbb{Q}[x]$ such that $N\left(f^{2}\right)<N(f)$.

In 1949 Erdős [6] proved that there exists an infinite sequence of polynomials $f_{n} \in \mathbb{Q}[x]$ such that $N\left(f_{n}\right) \rightarrow \infty$ and $N\left(f_{n}^{2}\right)<N\left(f_{n}\right)^{c}$, where $c<1$. The same year Verdenius [20] showed that one can take $c=\log 8 / \log 13 \approx 0.81 \ldots$

In 1991 D. Coppersmith and J. Davenport [2] proved that for every $l>1$ and a suitable sequence of polynomials $f_{n} \in \mathbb{Q}[x]$ with $N\left(f_{n}\right) \rightarrow \infty$ we have

$$
N\left(f_{n}^{l}\right) \ll N\left(f_{n}\right)^{c_{l}},
$$


where $c_{l}<1$ is effectively computable (unfortunately $c_{2}>\log 8 / \log 13$ ). Moreover they proved that for every $F \in \mathbb{C}[x], \partial F>1$ there exists an infinite sequence of polynomials $f_{n} \in \mathbb{C}[x]$ such that $N\left(f_{n}\right) \rightarrow \infty$ and

$$
N\left(F\left(f_{n}\right)\right) \ll N\left(f_{n}\right)^{c_{F}},
$$

where $c_{F}<1$

There remains the question whether for every sequence $f_{n}$ one can give a similar bound from below. In 1987 the author [16] gave the estimate

$$
N\left(f^{l}\right) \geq l+1+(\log 2)^{-1} \log \left(1+\frac{\log (N(f)-1)}{l \log 4 l-\log l}\right)
$$

for $l>1, f \in \mathbb{C}[x], N(f) \geq 2$. There is a great distance between the above upper and lower bounds for $N\left(f^{l}\right)$ already for $l=2$. Erdös thinks that his estimate is closer to the truth.

Perhaps for every non-constant $F$ the inequality holds

$$
N(F(f)) \geq \varphi_{F}(N(f)),
$$

for some function $\varphi_{F}$ satisfying the condition $\lim _{x \rightarrow \infty} \varphi_{F}(x)=\infty$.

Such function is unknown even for quite simple polynomials $F$, e.g. $F(x)=$ $x^{3}-x$.

We have begun the lecture from binomials, we shall finish with trinomials. Let us consider a field $K$ and a trinomial of the form

$$
x^{n}+A x^{m}+B, \quad \text { where } A, B \in K^{*} \text { and } n>m>0 .
$$

We observe that the trinomial is reducible over $K$ if and only if the trinomial $B x^{n}+A x^{n-m}+1$, or what comes to the same $x^{n}+A B^{-1} x^{n-m}+B^{-1}$ is reducible over $K$.

Since either $n \geq 2 m$, of $n \geq 2(n-m)$ we may assume without loss of generality that $n \geq 2 m$. Let us fix the following notation

$$
\operatorname{char} K:=\pi \geq 0, \quad n_{1}:=n /(n, m), \quad m_{1}:=n /(n, m)
$$

and let $\mathbf{y}$ denote a vector of independent variables. Assume that $\pi \nmid m n(n-m)$.

We shall state two theorems about reducibility of trinomials.

Theorem 1. Let $n \geq 2 m>0 ; A, B \in K(\mathbf{y})^{*}$, where $A^{-1} B^{n-m} \notin K$. The trinomial $x^{n}+A x^{m}+B$ is reducible over $K(\mathbf{y})$ if and only if either

(i) $x^{n}+A x^{m}+B$ has over $K(\mathbf{y})$ a proper factor of degree $\leq 2$

(ii) there exists an integer $l$ such that $\left\langle\frac{n}{l}, \frac{m}{l}\right\rangle:=\langle\nu, \mu\rangle \in S_{0}$, where

$$
S_{0}:=\prod_{p}\{\langle 2 p, p\rangle\} \cup\{\langle 6,1\rangle,\langle 6,2\rangle,\langle 7,1\rangle,\langle 8,2\rangle,\langle 8,4\rangle,\langle 9,3\rangle,
$$

$$
\langle 10,2\rangle,\langle 10,4\rangle,\langle 12,2\rangle,\langle 12,3\rangle,\langle 12,4\rangle,\langle 15,5\rangle\}
$$

and

$$
A=u^{\nu-\mu} A_{\nu, \mu}(v), \quad B=u^{\nu} B_{\nu \mu}(v)
$$


where $u, v \in K(\mathbf{y})$, $p$ runs over all primes and $A_{\nu, \mu}, B_{\nu, \mu}$ are given in Table 1 (below).

\section{Table 1}

\begin{tabular}{|c|c|c|}
\hline$\nu, \mu$ & $A_{\nu, \mu}$ & $B_{\nu, \mu}$ \\
\hline $2 p, p$ & $-\left(\frac{1+\sqrt{1-4 v}}{2}\right)^{p}-\left(\frac{1-\sqrt{1-4 v}}{2}\right)^{p}$ & $v^{p}$ \\
\hline 6,1 & $8 v\left(v^{2}+1\right)$ & $\left(v^{2}+4 v-1\right)\left(v^{2}-4 v-1\right)$ \\
\hline 6,2 & $4(v+1)$ & $-v^{2}$ \\
\hline 7,1 & $\begin{aligned}-(2 v+1)^{4}\left(4 v^{2}-\right. & 3 v+1) \\
& \times\left(v^{3}-2 v^{2}-v+1\right)\end{aligned}$ & $v(2 v-1)(2 v+1)^{5}(3 v-2)\left(v^{2}-v-1\right)$ \\
\hline 8,2 & $-v^{2}+8 v-8$ & $(2 v-2)^{2}$ \\
\hline 8,4 & $2 v^{2}-8 v+4$ & $v^{4}$ \\
\hline 9,3 & $v^{3}-81 v+243$ & $27(v-3)^{3}$ \\
\hline 10,2 & $4 v^{3}-8 v+4$ & $-\left(v^{2}-4 v+2\right)^{2}$ \\
\hline 10,4 & $v^{5}\left(-v^{3}+8 v-8\right)$ & $-4 v^{8}(v-1)^{4}$ \\
\hline 12,2 & $\begin{array}{r}1024(v-4)^{8}(2 v-3)\left(v^{2}-6 v+6\right) \\
\times\left(v^{2}-2 v+2\right)\end{array}$ & $1024(v-4)^{10}\left(v^{3}-8 v+8\right)^{2}$ \\
\hline 12,3 & $\begin{array}{r}-729 v(v-1)^{7}(2 v-1)\left(3 v^{2}-6 v+2\right) \\
\times\left(3 v^{2}-3 v+1\right)\end{array}$ & $729(v-1)^{9}\left(3 v^{3}-3 v+1\right)^{3}$ \\
\hline 12,4 & $512(2 v-1)\left(2 v^{2}+2 v-1\right)\left(2 v^{2}-2 v+1\right)$ & $1024\left(2 v^{2}-4 v+1\right)^{4}$ \\
\hline 15,5 & $\begin{array}{l}5(5 v-5)^{7}\left(5 v^{4}-5 v^{3}-5 v^{2}+5 v-1\right) \\
\quad \times\left(5 v^{4}-10 v^{3}+100 v^{2}-5 v+1\right)\end{array}$ & $(5 v-5)^{10}\left(5 v^{2}-5 v+1\right)^{5}$ \\
\hline
\end{tabular}

There is an analogy between this theorem and Capelli's theorem. There was one exceptional case there, here there are twelve. However Capelli's theorem is true over an arbitrary field, the above theorem only over an arbitrary field of rational functions.

Theorem 2. Let $n \geq 2 m>0,[K: \mathbb{Q}]<\infty ; A, B \in K^{*}$. The trinomial $x^{n}+A x^{m}+B$ is reducible over $K$ if and only if either one of the conditions (i), (ii) holds with $K$ in place of $K(\mathbf{y})$ or 
(iii) there exists an integer $l$ such that $\left\langle\frac{n}{l}, \frac{m}{l}\right\rangle:=\langle\nu, \mu\rangle \in S_{1}$, where

$$
S_{1}=\{\langle 7,2\rangle,\langle 7,3\rangle,\langle 8,1\rangle,\langle 9,1\rangle,\langle 14,2\rangle,\langle 21,7\rangle\}
$$

and $A=u^{\nu-\mu} A_{\nu, \mu}(v, w), B=u^{\nu} B_{\nu, \mu}(v, w)$, where $u \in K,\langle v, w\rangle \in E_{\nu, \mu}(K)$, while the polynomials $A_{\nu, \mu}, B_{\nu, \mu}$ and the elliptic curve $E_{\nu, \mu}$ are given in Table 2 (below) or

(iv) there exists an integer $l$ such that $\left\langle\frac{n}{l}, \frac{m}{l}\right\rangle:=\langle\nu, \mu\rangle \in \mathbb{Z}^{2}$ and $A=u^{\nu-\mu} A_{0}$, $B=u^{\nu} B_{0}, u \in K,\left\langle A_{0}, B_{0}\right\rangle \in F_{\nu, \mu}(K)$ and $F_{\nu, \mu}(K)$ is a certain finite (possibly empty) set.

Table 2

\begin{tabular}{|c|c|c|c|}
\hline$\nu, \mu$ & $E_{\nu, \mu}$ & $A_{\nu, \mu}$ & $B_{\nu, \mu}$ \\
\hline 7,2 & $\begin{array}{l}w^{2}=v^{3}+16 v^{2} \\
\quad+64 v+80\end{array}$ & $2 v^{2}-8 v-48+w(2 v-4)$ & $\begin{array}{l}-(4 v+12+w) \\
\quad \times\left(v^{2}+12 v+32+4 w\right)\end{array}$ \\
\hline 7,3 & $\begin{array}{l}w^{2}=v^{3}-675 v \\
\quad+13662\end{array}$ & $\begin{array}{l}\left(-v^{3}+27 v^{2}+3753 v\right. \\
\quad-34803+w(6 v-666)) \\
\quad \times(v-39)\end{array}$ & $\begin{array}{l}6(v-39)^{2}\left(-v^{2}-12 v\right. \\
\quad+693+6 w)\left(9 v^{2}+162 v\right. \\
\quad-4455-w(v+33))\end{array}$ \\
\hline \multirow{2}{*}{8,1} & $w^{2}=v^{3}-10 v+12$ & $\begin{array}{l}-8 v^{3}+20 v^{2}+8 v-32 \\
\quad+w\left(3 v^{2}-12 v-10\right)\end{array}$ & $\begin{array}{l}(w-3 v+5)\left(-3 v^{2}+15 v\right. \\
\quad-17+w(2 v-5))\end{array}$ \\
\hline & $w^{2}=v^{3}-20 v-16$ & $\begin{array}{l}128(w-2 v-8)^{4} \\
\quad \times(v+2)\left(v^{2}+12 v+4\right) \\
\quad \times\left(2 w-v^{2}+4 v+4\right) \\
\quad \times\left(4 w-v^{2}-12\right)\end{array}$ & $\begin{array}{l}64(w-2 v-8)^{4}\left(9 v^{4}+8 v^{3}\right. \\
\quad-8 v^{2}+288 v+272 \\
\left.\quad-w\left(v^{3}+18 v^{2}+76 v+24\right)\right) \\
\quad \times\left(v^{4}+24 v^{3}+152 v^{2}\right. \\
\quad+96 v+16+w\left(v^{3}-22 v^{2}\right. \\
\quad-52 v-72))\end{array}$ \\
\hline 9,1 & $w^{2}=v^{3}+18 v-36$ & $\begin{array}{l}81(w-2 v-9)^{4}\left(\left(v^{7}+27 v^{6}\right.\right. \\
\quad+351 v^{5}+639 v^{4}-675 v^{3} \\
\quad-5589 v^{2}+6318 v \\
\quad-7290) w+\left(-9 v^{8}-66 v^{7}\right. \\
\quad-936 v^{6}+1890 v^{5}+4995 v^{4} \\
\quad-5670 v^{3}+14580 v^{2} \\
\quad-72900 v+37179))\end{array}$ & $\begin{array}{l}27(w-2 v-9)^{5}\left(\left(5 v^{7}-603 v^{6}\right.\right. \\
\quad-765 v^{5}+5661 v^{4}+3213 v^{3} \\
\quad+29889 v^{2}-28674 v \\
\quad+10206) w+\left(-v^{9}+63 v^{8}\right. \\
\quad+1719 v^{7}-4959 v^{6} \\
\quad-10611 v^{5}+1917 v^{4} \\
\quad+111456 v^{3}-145800 v^{2} \\
\quad+207036 v-61236))\end{array}$ \\
\hline
\end{tabular}


Table 2 continued

\begin{tabular}{|c|c|c|c|}
\hline$\nu, \mu$ & $E_{\nu, \mu}$ & $A_{\nu, \mu}$ & $B_{\nu, \mu}$ \\
\hline 14,2 & $w^{2}=v^{3}-6 v+5$ & $\begin{array}{c}4(v-2)^{7}\left(4 v^{4}-v^{3}\right. \\
-34 v^{2}+51 v-18 \\
\left.+w\left(v^{3}+6 v^{2}-18 v+8\right)\right)\end{array}$ & $\begin{array}{c}+(v-2)^{8}\left(v^{3}-12 v+14\right. \\
+w(2 v-6))^{2}\end{array}$ \\
\hline
\end{tabular}

$\nu=21, \mu=7$

$E_{\nu, \mu}: \quad w^{2}=v^{3}-1715 v+33614$

$A_{\nu, \mu}=3764768(w-7 v-343)^{7} \times\left(-70 v^{13}-52822 v^{12}+19467098 v^{11}+3451790790 v^{10}\right.$

$-68568103744 v^{9}-7533659832748 v^{8}+155066962439572 v^{7}+6992189738638860 v^{6}$

$+111845300294417242 v^{5}-2615541950886590670 v^{4}-185207197444036469646 v^{3}$

$-2167406145663758747314 v^{2}-17859482834686233287988 v$

$-18838244084537504480336) w+\left(v^{15}+2625 v^{4}+91584 v^{13}-411648706 v^{12}\right.$

$-8059651761 v^{11}+1191725696763 v^{10}+27401291878562 v^{9}-2107086579531888 v^{8}$

$-82212564592345537 v^{7}+2560864878174600039 v^{6}+64436612556278953228 v^{5}$

$-653044731700569035282 v^{4}-20619925798094466268271 v^{3}$

- $399648258921266894946883 v^{2}-1749201525015966507411086 v$

$-9642297897576373802186512)$.

$B_{\nu, \mu}=14^{7}(w-7 v-343)^{14}\left(21 v^{2}-686 v-7203-(v+49) w\right)^{7}$

As to Table 2 the curve $E_{7,2}$ is not in Weierstrass normal form, since to have it reduced would require char $K \neq 3$. In the case $\langle\nu, \mu\rangle=\langle 8,1\rangle$ we have a double choice. The polynomial $A_{21,7}$ has been computed by Prof. J. Browkin using the programme GP-PARI, some other polynomials by Dr. A. Pokrzywa, using the programme Mathematica.

Table 3. Sporadic trinomials over $\mathbb{Q}$

The table contains all reducible trinomials $x^{n}+A x^{m}+B, n \geq 2 m, A, B \in \mathbb{Z} \backslash\{0\}$ known to the author, which satisfy neither (vi) nor (vii) nor (viii) and have the following properties: 1) for every greater than 1 divisor $d$ of $(n, m) x^{n / d}+A x^{m / d}+B$ is irreducible, 2) $\left(A^{n}, B^{n-m}\right)$ is free from $n(n-m)$ th powers, 3$)$ if $n-m$ is odd then $A>0$, if $n, m$ both odd, then $B>0$.

\begin{tabular}{|c|c|c|l|}
\hline Number & Trinomial & Factor & Discoverer \\
\hline 1 & $x^{8}+3 x^{3}-1$ & $x^{3}+x-1$ & Eutczyk \\
\hline 2 & $x^{8}+2^{3} \cdot 3 x^{3}+2^{5}$ & $x^{3}-2 x^{2}+4$ & Nicolas \\
\hline 3 & $x^{8}+2^{2} \cdot 3^{3} x^{3}+3^{5}$ & $x^{3}+3 x^{2}+9 x+9$ & Nicolas \\
\hline 4 & $x^{8}+3 \cdot 5 \cdot 7^{3} \cdot 59 x^{3}-2^{3} \cdot 7^{5} \cdot 11^{3}$ & $x^{3}-7 x^{2}-98 x+2156$ & Author \\
\hline 5 & $x^{9}-2^{2} \cdot 19 x+2^{5} \cdot 3$ & $x^{4}-2 x^{2}-4 x+6$ & Author \\
\hline 6 & $x^{9}+2^{5} x^{2}-2^{6}$ & $x^{3}-2 x^{2}+4 x-4$ & Nicolas \\
\hline
\end{tabular}


Table 3 continued

\begin{tabular}{|c|c|c|c|}
\hline Number & Trinomial & Factor & Discoverer \\
\hline 7 & $x^{9}+3^{4} x^{2}-2 \cdot 3^{3}$ & $x^{3}+3 x+3$ & Nicolas \\
\hline 8 & $x^{9}+3^{6} x^{2}-2 \cdot 3^{6}$ & $x^{3}-3 x^{2}+9$ & Browkin \\
\hline 9 & $x^{9}+3^{5} x^{4}+2^{2} \cdot 3^{6}$ & $x^{3}-3 x^{2}+18$ & Browkin \\
\hline 10 & $x^{9}+2^{4} \cdot 3^{5} x^{4}-2^{8} \cdot 3^{6}$ & $x^{3}+6 x^{2}+36 x+72$ & Nicolas \\
\hline 11 & $x^{10}+3^{3} \cdot 11 x-3^{5}$ & $x^{3}+3 x-3$ & Author \\
\hline 12 & $\begin{array}{r}x^{10}+2^{6} \cdot 3^{3} \cdot 5^{6} \cdot 11 x \\
-2^{7} \cdot 3^{5} \cdot 5^{5} \cdot 19\end{array}$ & $x^{4}-60 x^{2}-300 x+5400$ & Browkin \\
\hline 13 & $x^{10}+3 x^{3}-2^{3}$ & $x^{4}+x^{3}-x-2$ & Morain \\
\hline 14 & $x^{10}+2^{5} x^{3}-2^{6}$ & $x^{5}-2 x^{4}+8 x-8$ & Morain \\
\hline 15 & $x^{10}+3^{2} \cdot 11 x^{3}+2 \cdot 3^{3}$ & $x^{3}+3 x+3$ & Nicolas \\
\hline 16 & $x^{11}+2^{2} \cdot 3 x+2^{3}$ & $x^{5}-2 x^{4}+2 x^{3}-2 x^{2}+2$ & Nicolas \\
\hline 17 & $x^{11}+2^{3} \cdot 3^{3} \cdot 23 x^{2}-2^{4} \cdot 3^{5}$ & $x^{3}+6 x-6$ & Browkin \\
\hline 18 & $x^{11}+2^{2} \cdot 23 x^{3}+2^{3} \cdot 3$ & $x^{3}+2 x^{2}+4 x+2$ & Morain \\
\hline 19 & $x^{11}+x^{4}+2^{2}$ & $x^{5}-x^{3}-x^{2}+2$ & Jonassen \\
\hline 20 & $x^{11}-3^{3} \cdot 5^{2} \cdot 23 x^{5}+3^{8} \cdot 5^{4}$ & $x^{3}-15 x-45$ & Browkin \\
\hline 21 & $x^{12}+2^{6} \cdot 3^{2} x+2^{4} \cdot 23$ & $x^{3}+2 x^{2}+4 x+2$ & $\begin{array}{l}\text { Browkin-- } \\
\text { Author }\end{array}$ \\
\hline 22 & $x^{12}+2^{5} \cdot 3^{4} \cdot 13 x+2^{4} \cdot 3^{4} \cdot 23$ & $x^{3}+6 x+6$ & Browkin \\
\hline 23 & $x^{12}+2^{6} x^{5}-2^{8}$ & $x^{3}-2 x^{2}+4 x-4$ & Morain \\
\hline 24 & $x^{13}+2^{8} \cdot 3 x+2^{10}$ & $x^{3}+2 x^{2}+4 x+4$ & Browkin \\
\hline 25 & $x^{13}+2^{8} \cdot 3 \cdot 53 x-2^{12} \cdot 7$ & $x^{3}-4 x^{2}+8 x-4$ & $\begin{array}{l}\text { Browkin-- } \\
\text { Author }\end{array}$ \\
\hline 26 & $\begin{aligned} x^{13}+2^{8} \cdot 3 \cdot 5^{6} \cdot 53 x \\
+2^{11} \cdot 5^{7} \cdot 13\end{aligned}$ & $x^{3}+20 x+100$ & Browkin \\
\hline 27 & $\begin{array}{r}x^{13}-2^{6} \cdot 3 \cdot 5^{5} \cdot 53 x^{3} \\
+2^{8} \cdot 5^{8} \cdot 11\end{array}$ & $x^{3}+20 x-100$ & Browkin \\
\hline 28 & $x^{13}+3 x^{4}-1$ & $x^{3}+x^{2}-1$ & Coray \\
\hline
\end{tabular}


Table 3 continued

\begin{tabular}{|c|c|c|c|}
\hline Number & Trinomial & Factor & Discoverer \\
\hline 29 & $x^{13}+2^{6} \cdot 3 x^{4}-2^{9}$ & $x^{3}+2 x^{2}+4 x+4$ & Browkin \\
\hline 30 & $x^{13}+3^{3} \cdot 53 x^{4}-2^{2} \cdot 3^{6}$ & $x^{3}-3 x^{2}+6$ & Browkin \\
\hline 31 & $x^{13}+3 x^{6}+1$ & $x^{4}-x+1$ & Coray \\
\hline 32 & $x^{13}+2^{4} \cdot 3 x^{6}-2^{8}$ & $x^{3}-2 x^{2}+4 x-4$ & Browkin \\
\hline 33 & $x^{14}+2^{2} x+3$ & $x^{3}-x^{2}+1$ & Bremner \\
\hline 34 & $x^{14}+2^{2} x^{5}-1$ & $x^{3}+x^{2}-1$ & Bremner \\
\hline 35 & $x^{14}+2^{2} \cdot 3^{6} x^{5}+3^{11}$ & $x^{4}-3 x^{3}+9 x^{2}-18 x+27$ & Morain \\
\hline 36 & $x^{15}-3^{7} \cdot 5^{6} \cdot 31 x+2^{2} \cdot 3^{8} \cdot 5^{5} \cdot 29$ & $x^{3}+15 x-45$ & Browkin \\
\hline 37 & $x^{15}-2^{4} \cdot 7^{3} \cdot 31 x^{7}+2^{11} \cdot 3 \cdot 7^{5}$ & $x^{3}-14 x-28$ & Browkin \\
\hline 38 & $x^{16}+7 x^{3}+3$ & $x^{3}-x^{2}+1$ & Bremner \\
\hline 39 & $x^{16}+2^{3} \cdot 7 x^{3}-3^{2}$ & $x^{3}+x^{2}+x-1$ & Bremner \\
\hline 40 & $x^{16}+2^{8} x^{7}+2^{12}$ & $x^{4}-2 x^{3}+4 x^{2}-8 x+8$ & Morain \\
\hline 41 & $x^{16}+2^{8} \cdot 7 x^{7}-2^{15}$ & $x^{3}+2 x^{2}-8$ & Bremner \\
\hline 42 & $x^{17}+103 x+2^{3} \cdot 7$ & $x^{3}-x^{2}+x+1$ & Bremner \\
\hline 43 & $x^{17}+2^{12} \cdot 103 x^{4}-2^{16} \cdot 3^{2}$ & $x^{3}+2 x^{2}+4 x-8$ & Browkin \\
\hline 44 & $x^{21}+2^{11} \cdot 13 x^{5}+2^{14} \cdot 3$ & $x^{3}-2 x^{2}+4$ & Browkin \\
\hline 45 & $x^{22}+2^{14} \cdot 23 x-2^{15} \cdot 13$ & $x^{3}+2 x^{2}-4$ & Browkin \\
\hline 46 & $x^{24}+2^{11} \cdot 7 x+2^{8} \cdot 47$ & $x^{3}-2 x^{2}+2$ & $\begin{array}{c}\text { Browkin- } \\
\text { Author }\end{array}$ \\
\hline 47 & $x^{26}+2^{7} \cdot 3 \cdot 53 x^{3}+2^{8} \cdot 47$ & $x^{3}-2 x^{2}+2$ & $\begin{array}{l}\text { Browkin- } \\
\text { Author }\end{array}$ \\
\hline 48 & $x^{33}+67 x^{11}+1$ & $x^{3}+x+1$ & Bremner \\
\hline 49 & $x^{39}+2^{9} \cdot 3 \cdot 157 x^{13}+2^{13}$ & $x^{3}+2 x+2$ & Browkin \\
\hline 50 & $x^{46}+2^{26} \cdot 47 x^{7}-2^{31} \cdot 3^{2}$ & $x^{3}-2 x^{2}+4 x-4$ & Browkin \\
\hline 51 & $x^{51}-2^{31} \cdot 103 x^{5}+2^{34} \cdot 47$ & $x^{3}-2 x^{2}+4 x-4$ & Browkin \\
\hline 52 & $x^{52}+2^{34} \cdot 3 \cdot 53 x+2^{35} \cdot 103$ & $x^{3}+2 x^{2}+4 x+4$ & Browkin \\
\hline
\end{tabular}


Table 3 shows 52 reducible trinomials over $\mathbb{Q}$ that do not satisfy (i)-(iii). We propose

Conjecture. For every algebraic number field $K$ sets $F_{\nu, \mu}(K)$ can be chosen so that the set

is finite.

$$
\bigcup_{\langle\nu, \mu\rangle} \bigcup_{\left\langle A_{0}, B_{0}\right\rangle \in F_{\nu, \mu}(K)}\left\{x^{\nu}+A_{0} x^{\mu}+B_{0}\right\}
$$

For $K=\mathbb{Q}$ the conjecture implies that Table 3 cannot be indefinitely extended. To disprove the conjecture over $\mathbb{Q}$ seems very hard. In particular, author's results from [14] show that for every pair $\langle a, b\rangle \in \mathbb{Q}^{2}$ there exist only finitely many trinomials $x^{n}+a x^{m}+b$ reducible, but not satisfying (i), while the recent results of Györy and the author [8] show that for every fixed polynomial $f$ there exist only finitely many trinomials over $\mathbb{Q}$ divisible by $f$, but satisfying neither (i) nor (ii).

Finally, we shall note two simple consequences of the conjecture.

CONSEQUence 1 . For every algebraic number field $K$ there exists a constant $C_{1}(K)$ such that, if $n>2 m, A, B \in K^{*}$ and the trinomial $x^{n}+A x^{m}+B$ is reducible over $K$ then either $x^{n_{1}}+A x^{m_{1}}+B$ has a proper factor of degree $\leq 2$ or $n_{1} \leq C_{1}(K)$.

Remark. For $K=\mathbb{Q}$ we have $C_{1}(\mathbb{Q}) \geq 52$.

CONSEQUENCE 2. For every algebraic number field $K$ there exists a constant $C_{2}(K)$ such that every trinomial over $K$ has at least one irreducible factor with at most $C_{2}(K)$ non-zero coefficients.

Remark. For $K=\mathbb{Q}$ we have $C_{2}(\mathbb{Q}) \geq 8$.

\section{References}

[1] A. Capelli, Sulla riduttibilita delle equazioni algebriche, Nota prima, Rend. Accad. Fis. Mat. Soc. Napoli (3), 3 (1897), 243-252.

[2] D. Coppersmith and J. Davenport, Polynomials whose powers are sparse, Acta Arith. 58 (1991), 79-87.

[3] E. Dobrowolski, On a question of Lehmer and the number of irreducible factors of a polynomial, Acta Arith. 34 (1979), 391-401.

[4] F. Dorey and G. Whaples, Prime and composite polynomials, J. Algebra 28 (1974), 88-101.

[5] A. Dubickas, On a conjecture of A. Schinzel and H. Zassenhaus, Acta Arith. 63 (1993), $15-20$.

[6] P. Erdös, On the number of terms of the square of a polynomial, Nieuw. Arch. Wiskunde (2) 23 (1949), 63-65.

[7] M. Fried and R. E. MacRae, On the invariance of the chain of fields, Illinois J. Math. 13 (1969), 165-171.

[8] K. Győry and A. Schinzel, On a conjecture of Posner and Rumsey, J. Number Theory 47 (1994), 63-78. 
[9] D. H. Lehmer, Factorization of certain cyclotomic functions, Ann. of Math. 34 (1933), 461-479.

[10] R. Louboutin, Sur la mesure de Mahler d'un nombre algébrique, C. R. Acad. Sci. Paris, Sér. I 296 (1983), 539-542.

[11] L. Rédei, Algebra, Erster Teil, Akademische Verlaggesellschaft, Leipzig, 1959.

[12] A. Rényi, On the minimal number of terms in the square of a polynomial, Hungar. Acta Math. 1 (1947), 30-34 = Selected papers, vol. 1, Budapest 1976, 42-47.

[13] J. F. Ritt, Prime and composite polynomials, Trans. Amer. Math. Soc., 23 (1922), $51-66$.

[14] A. Schinzel, Reducibility of lacunary polynomials I, Acta Arith. 16 (1969), 123-159.

[15] A. Schinzel, Selected topics on polynomials, The University of Michigan Press, Ann Arbor, 1982.

[16] A. Schinzel, On the number of terms of a power of a polynomial, Acta Arith. 49 (1987), 55-70.

[17] A. Schinzel, On reducible trinomials, Dissertationes Math. 329 (1993).

[18] A. Schinzel and H. Zassenhaus, A refinement of two theorems of Kronecker, Michigan Math. J. 12 (1965), 81-85.

[19] C. J. Smyth, On the product of the conjugates outside the unit circle of an algebraic integer, Bull. London Math. Soc. 3 (1971), 169-175.

[20] W. Verdenius, On the number of terms of the square and the cube of polynomials, Indag. Math. 11 (1949), 459-465.

[21] U. Zannier, Ritt's second theorem for arbitrary characteristic, J. Reine Angew. Math. 445 (1993), 175-203. 